\title{
Curative effects of head $\gamma$-SRT for the treatment of functional pituitary macroadenoma
}

\author{
WEI LIAN, REN ZHI WANG, BING XING and YONG YAO \\ Department of Neurosurgery, Peking Union Medical College Hospital, Chinese Academy of \\ Medical Sciences and Peking Union Medical College, Beijing 100730, P.R. China
}

Received January 25, 2016; Accepted June 14, 2016

DOI: $10.3892 / \mathrm{ol} .2016 .4712$

\begin{abstract}
The aim of the study was to examine the curative effects and proper radiotherapy plan of head $\gamma$-stereotactic radiotherapy $(\gamma$-SRT) for the treatment of functional pituitary macroadenoma. Clinical samples of 30 patients that underwent $\gamma$-SRT (radiotherapy group) and 26 patients that underwent pituitary adenoma resection via single nasal-sphenoidal approach (surgery group) were analyzed retrospectively and their curative effects were compared. The results showed that in the radiotherapy group, 12 cases accepted single fraction irradiation, with an average maximum diameter of tumor body of $1.8 \pm 0.6 \mathrm{~cm}$, average volume of $0.6 \pm 0.4 \mathrm{~cm}^{3}$, average dose of the central point of $52.6 \pm 18.7 \mathrm{~Gy}$, average dose of the peripheral point of $24.7 \pm 10.2 \mathrm{mGy}$, and isodose curve of $50-70 \%$. The remaining 18 cases accepted multiple fraction irradiation, with an average irradiation of $3.7 \pm 1.6$ times, maximum average diameter of tumor body of $4.3 \pm 1.8 \mathrm{~cm}$, average volume of $4.8 \pm 2.7 \mathrm{~cm}^{3}$, average dose of the central point of $24.6 \pm 12.5 \mathrm{~Gy}$,

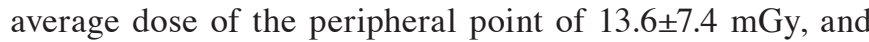
isodose curve of $50-70 \%$. Following treatment, the tumor volumes of patients in the radiotherapy group that received single and multiple irradiation were significantly reduced and the visual acuity and visual field were improved $(\mathrm{p}<0.05)$. The two groups were followed up for an average of 3.8 years, and the follow-up results showed that differences of the two groups on the tumor control, mortality and hypopituitarism rates were not statistically significant ( $p>0.05$ ). In addition, the incidence of complications of the radiotherapy group was significantly decreased as compared to that of the surgery group $(\mathrm{p}<0.05)$. In conclusion, $\gamma$-SRT was safe and effective for the treatment of functional pituitary adenomas. Its curative effects were
\end{abstract}

Correspondence to: Dr Wei Lian, Department of Neurosurgery, Peking Union Medical College Hospital, Chinese Academy of Medical Sciences and Peking Union Medical College, 1 Shuai-Fu-Yuan Hutong, Beijing 100730, P.R. China

E-mail: rrm062@163.com

Key words: $\gamma$-stereotactic radiotherapy, functional pituitary macroadenoma, pituitary adenoma resection, single nasal-sphenoidal approach equivalent to that of the microscopic single nasal-sphenoidal approach with fewer complications.

\section{Introduction}

Pituitary tumor is the most common intracranial primary endocrine tumor, accounting for approximately 20-25\% (1). According to the biological characteristics, the pituitary tumor can be divided into functional and non-functional pituitary tumor. The ideal target of the tumor is to control the volume, reduce its stress effects on peripheral tissues and keep the endocrine hormones within a normal range. $\gamma$-stereotactic radiotherapy $(\gamma$-SRT), based on traditional two-dimensional radiotherapy and combined with the advantages of the gamma knife, X-knife and CyberKnife in the head-position (whole body), such as high energy, high precision, high efficacy and low injury, has been widely used in intracranial tumor, lung tumor, mediastinal tumor, liver tumor and pelvic tumor (2).

Previous findings suggest that single or multiple SRT is superior to surgical treatment in small non-functional pituitary tumors (3). However, its irradiation regimens, treatment effects and complications in functional acroadenoma remain to be determined. Scholars tend to take it as adjuvant or palliative therapy (4). Other scholars observed that SRT was safe and effective in pituitary macroadenoma (5), but due to a limited sample size, its effects for functional adenoma were not further analyzed. In addition, microscopic pituitary adenoma resection via the single nasal-sphenoidal approach can improve the tumor resection rate and reduce complications and has become increasingly utilized (6).

The aim of the study was to examine the appropriate treatment regimen for functional pituitary macroadenoma by comparing and analyzing the $\gamma$-SRT and single nasal-sphenoidal approach.

\section{Materials and methods}

Materials. In total, 63 patients diagnosed with functional pituitary tumor at the Chinese Academy of Medical Sciences and Peking Union Medical College (Beijing, China) between March 2010 and March 2015 were included in the study. Following CT or MRI examination, endocrine hormone and pathological diagnosis, the patients were confirmed as benign 
adenoma. Inclusion criteria for the study were: i) tumor diameter $>1 \mathrm{~cm}$; ii) ineffective drug therapy; and iii) first treatment, complete clinical data. Exclusion criteria for the study were: i) giant pituitary adenoma, accompanied with severe visual acuity and visual field impairment, hydrocephalus and increased intracranial pressure; ii) other intracranial tumors such as meningioma, pituitary target organ tumors, such as adrenal and gonadal tumors; and iii) interruptive treatment or lost follow-up.

Approval for the study was obtained from the Ethics Committee of the Chinese Academy of Medical Sciences and Peking Union Medical College. Informed consent was provided by patients and their families. According to the treatment methods, the patients were divided into the radiotherapy $(n=33)$ and surgery $(n=30)$ groups. Three cases from the radiotherapy group and 4 cases from the surgery group were lost to follow up. Additionally, the radiotherapy group comprised 30 cases and the surgery group, 26 cases. Patients in the radiotherapy group underwent head $\gamma$-SRT treatment whereas patients in the surgery group underwent the microscopic single nasal-sphenoidal approach. The radiotherapy group included 17 men and 13 women, with a range of 32-65 years and an average of $47.8 \pm 12.3$ years; 12 cases of prolactin (PRL) tumor, 9 cases of growth hormone $(\mathrm{GH})$ tumor, 5 cases of adrenocorticotropic hormone (ACTH) tumor, and 4 cases of thyroid-stimulating hormone (TSH) tumor. According to the Hardy typing standard there were 15 cases of localized II, 10 cases of invasive III and 5 cases of invasive IV, with a maximum tumor diameter of $1.5-5.0 \mathrm{~cm}$ and an average of $3.3 \pm 1.7 \mathrm{~cm}$. The surgery group included 16 men and 10 women, with a range of 34-68 years and an average of $48.2 \pm 14.5$ years; 13 cases of PRL tumor, 8 cases of GH tumor, 3 cases of ACTH tumor, and 2 cases of TSH tumor. According to the Hardy typing standard there were 12 cases of localized II, 9 cases of invasive III and 5 cases of invasive IV; with a maximum tumor diameter of $1.5-5.5 \mathrm{~cm}$ and an average of $3.5 \pm 1.9 \mathrm{~cm}$. Differences of the two groups on gender, age, tumor classification, typing and diameter were not statistically significant $(\mathrm{p}>0.05)$.

\section{Methods}

\section{Head $\gamma$-SRT procedures}

Installation of stereo positioning frame. Metal and magnetic objects that potentially interfered with the magnetic resonance images were removed. A line was made at $2.0 \mathrm{~cm}$ above the outer canthus and $3.5 \mathrm{~cm}$ above the center of the external auditory foramen as the surface marker of anterior/posterior commissure (ACPC) line, the basic ring of the Leksell C type stereotactic frame (Elekta Instruments AB, Stockholm, Sweden) was kept parallel to the ACPC line. The scalp was disinfected and local anesthesia was carried out using lidocaine, by fixing the frame onto the external plate of the skull.

Magnetic resonance scanning. Axial, coronal and sagittal scanning was performed using a Magnetom Vision type $1.5 \mathrm{~T}$ high field strength magnetic resonance machine (Siemens, Erlangen, Germany), with a layer thickness of 2-3 mm, and space free. According to the condition of disease, T1WISag, T1WICor, T2WICor or enhanced T1WISag, T1WICor and dynamic scan was selected. The lesion and its peripheral structures were scanned, and alteration of the scan parameter and site according to the images of lesion and adjacent structures was determined.

Therapeutic process. The magnetic resonance required by the gamma knife to scan was selected and the collected axial was located and coronal magnetic resonance images of the pituitary tumor, optic nerve and eyeball plane were performed. The images were scanned using a USB Scanner Astra 4000U scanner (512x512 matrix) in the computer image acquisition system to fix the position, normalize, sequence and process helmet parameters according to the manufacturer's instructions for SGI Indy 3D graphics workstation version 2.1 software (Silicon Graphics Inc., SanFrancisco, CA, USA). A $4 \mathrm{~mm}$ collimator, 1-10 treatment targets, according to the maximum range of exposure dose that different tissues were able to tolerate, optic nerve $<10 \mathrm{~Gy}$, brain stem $<15 \mathrm{~Gy}$, peripheral cranial nerve $<13-18$ Gy, target center dose of 24-50 Gy, changed $\gamma$ angle and used shield block were employed to protect the patient crystalline lens and optic nerve. The treatment plan was checked and the patients were sent into the machine room, and $\mathrm{x}, \mathrm{y}$ and $\mathrm{z}$ three-dimensional coordinates were adjusted, followed by turning on the cobalt bomb and commencing the $\gamma$-SRT. The tumor was completely covered and enveloped by a small collimator and multi-target, selected $72^{\circ} \gamma$ angle. Gun points were 7.7 for $4 \mathrm{~mm}$ microadenoma, 1 for $8 \mathrm{~mm}$ microadenoma, 9 for $4 \mathrm{~mm}$ macroadenoma, 6.9 for $8 \mathrm{~mm}$ macroadenoma and 0.6 for $14 \mathrm{~mm}$ macroadenoma. The computer automatically set the target of the same collimator at the same RUN. Automatic position system (APS) automatically performed the irradiation of each target after reviewing the RUN that needed irradiation.

Microscopic pituitary adenoma resection via the single nasal-sphenoidal approach. The patient was maintained in a supine position and the head was fixed onto a Mayfield head supporter (Integra; Garland, TX, USA), approached via the left nostril and dilated using the nose dilation instrument to disconnect the bony part of nasal septum. Under a microscope (Leica, Germany), the nasal mucosa was carefully separated to expose the opening of sphenoid sinus, and an osteotome was used to chisel and open the anterior sphenoidal wall along the midline to expose the sellar floor. The procedure was performed with full attention by avoiding touching of the optic nerve groove and carotid artery pressure trace, and the tissues of the sellar bottom were chiseled and opened according to the midline position, the endocranium was exposed and cut open using the ' $\dagger$ ' method. Full attention was paid not to exceed the interface of the diaphragma sellae and dura mater of the frontal base. A curet was used to collect samples for biopsy, and an aspirator was used to absorb the pituitary tumor body, which was filled in with gelatin sponge and autologous muscle.

Observation index. Tumor volume, tumor control rate, improvement rate of visual acuity and visual field, incidence of pituitary function and complication were compared. Pituitary MRI, pituitary function (endocrine hormone) and visual acuity and visual field were re-examined every 3 months in the first year after treatment, and re-examined every 6 months after the first year. 
Statistical analysis. The SPSS 20.0 statistical software (IBM, Armonk, NY, USA) was used for statistical analysis. Data were presented as mean \pm standard deviation and the group comparison was made using a Student's t-test. Countable data were expressed as a percentage (\%) and the group comparison was made using an $\chi^{2}$ test. $\mathrm{P}<0.05$ was considered statistically significant.

\section{Results}

Comparison of tumor volume, visual acuity and visual field Radiotherapy group. Twelve cases of single fraction irradiation were accepted, the maximum diameter of the tumor body was $1.5-2.0 \mathrm{~cm}$ and an average of $1.8 \pm 0.6 \mathrm{~cm}$; tumor volume $(\mathrm{V}=4 / 3 \mathrm{x}$ maximum diameter $/ 2 \mathrm{x}$ minimum diameter $/ 2 \mathrm{x}$ height $/ 2$ ) was $0.5-1 \mathrm{~cm}^{3}$, with an average of $0.6 \pm 0.4 \mathrm{~cm}^{3}$; dose of the central point was $23-5 \mathrm{~Gy}$, with an average dose of $52.6 \pm 18.7 \mathrm{~Gy}$; dose of the peripheral point was $10-35 \mathrm{~Gy}$, with an average of $24.7 \pm 10.2 \mathrm{mGy}$; and the isodose curve was $50-70 \%$. Prior to treatment, visual acuity was $0.3-0.6$, average $0.4 \pm 0.3$; temporal defect in 10 cases, ranging from 70 to $85^{\circ}$, average $80.5 \pm 16.4^{\circ}$; and nasal defect in 2 cases, ranging from 50 to $55^{\circ}$. Following treatment, the tumor volume was $0.05-0.2 \mathrm{~cm}^{3}$, average $0.08 \pm 0.03 \mathrm{~cm}^{3}$; visual acuity was $0.5-0.8$, average $0.7 \pm 0.4$; and the temporal side of visual field was $80-93^{\circ}$, average $86.7 \pm 18.2^{\circ}$.

The remaining 18 cases accepted multiple fraction irradiation from 2 to 5 times, average $3.7 \pm 1.6$ times; the maximum diameter of the tumor body was $2.2-5.0 \mathrm{~cm}$, average $4.3 \pm 1.8 \mathrm{~cm}$; tumor volume was $0.8-7.3 \mathrm{~cm}^{3}$, average $4.8 \pm 2.7 \mathrm{~cm}^{3}$; dose of the central point was $12-35 \mathrm{~Gy}$, average $24.6 \pm 12.5 \mathrm{~Gy}$; dose of the peripheral point was 5-30 Gy, average 13.6 $\pm 7.4 \mathrm{mGy}$; and the isodose curve was $50-70 \%$. Prior to treatment, the visual acuity was $0.3-0.5$, average $0.4 \pm 0.7$; temporal defect in 15 cases, ranged from 65 to $80^{\circ}$, average $72.8 \pm 19.3^{\circ}$; and nasal defect was identified in 3 cases, ranging from 45 to $50^{\circ}$. Following treatment, the tumor volume was $0.2-1.3 \mathrm{~cm}^{3}$, average $0.7 \pm 0.4 \mathrm{~cm}^{3}$; visual acuity was $0.5-0.7$, average $0.6 \pm 0.3$; and the temporal side of the visual field was $75-90^{\circ}$, average $183.4 \pm 15.5^{\circ}$. Following treatment, the tumor volumes of patients in the radiotherapy group that received single and multiple irradiation were significantly reduced, and the visual acuity and visual field were improved, with differences being statistically significant $(\mathrm{p}<0.05)$.

Surgery group. Before treatment, visual acuity was 0.3-0.7, average $0.5 \pm 0.4$; tumor volume was $0.4-7.1 \mathrm{~cm}^{3}$, average $3.5 \pm 2.2 \mathrm{~cm}^{3}$; temporal defect was identified in 14 cases, ranging from 68 to $82^{\circ}$, average $75.6 \pm 18.2^{\circ}$; nasal defect was identified in 4 cases, ranging from 46 to $60^{\circ}$. Total resection was performed in 7 cases, a subtotal resection in 8 cases, and a partial resection in 3 cases. Following treatment, tumor volume was $0.02-0.8 \mathrm{~cm}^{3}$, average $0.3 \pm 0.7 \mathrm{~cm}^{3}$; visual acuity was $0.5-0.8$, average $0.6 \pm 0.4$, temporal side of visual field was $78-93^{\circ}$, average $86.7 \pm 13.4^{\circ}$. Additionally, the tumor volumes of patients in the surgery group were significantly reduced, visual acuity and visual field were improved, and the differences were statistically significant $(\mathrm{p}<0.05)$.

Differences in tumor volume, improvement of visual acuity and visual field of the two groups before and after treatment were not statistically significant ( $\mathrm{p}>0.05$ ). The radiotherapy group was followed up for 6-5.3 months, average 3.8 years; while the surgery group was followed up for 6-5.6 months, average 3.9 years. The difference on follow-up time of the two groups was not statistically significant ( $p>0.05$ ). For the radiotherapy group, tumor stabilization was identified in 23 cases, tumor progression in 4 cases and death in 3 cases (10\%). For the surgery group, tumor stabilization was identified in 18 cases, tumor progression in 5 cases and death in 3 cases $(11.5 \%)$. Difference in tumor control and mortality rates of the two groups was not statistically significant $(\mathrm{p}>0.05)$.

Comparison of pituitary functions and complications. After hormone replacement therapy, 3 cases of the radiotherapy group under single irradiation and 2 cases under multiple irradiation suffered persistent hypopituitarism and the reduction rate was $16.7 \%$. In the surgery group, 3 cases (11.5\%) suffered hypopituitarism, and the difference of the two groups on pituitary decline rate was not statistically significant $(p>0.05)$. The main complications of radiotherapy group were hypopituitarism, visual acuity and visual filed deterioration (cranial nerve injury), hypothalamic injury, and secondary tumor induction. The main complications for the surgery group were cerebrospinal rhinorrhea, hypopituitarism (mostly diabetes diabetes insipidus), water and sodium disturbance, hemorrhage and infection. The incidence of complications for the radiotherapy group was significantly lower than that of the surgery group ( 16.7 vs. $\left.42.3 \%, \chi^{2}=4.487, \mathrm{p}=0.034\right)$.

\section{Discussion}

Since the Swedish neurosurgeon Leksell suggested the concept of stereotactic radiosurgery in 1951 and initially used the gamma knife in the treatment of pituitary tumor in 1968 (7), the clinical effects of this knife for pituitary tumor treatment has been investigated. Its indications include: microadenoma with diameter $<1 \mathrm{~cm}$; macroadenoma with diameter $>1 \mathrm{~cm}$, without obvious visual acuity and visual field damages, with tumor being $\geq 2 \mathrm{~mm}$ away from the optic chiasma; patients with tumor residues or recurrence; and elderly patients or patients unable to tolerate surgery (8). Other scholars have studied the effects of the gamma knife for the giant pituitary tumor. Weber et al retrospectively analyzed the data of 27 patients that received fractionated SRT treatment (FSRT) (9), including 10 cases of functional adenoma and 17 cases of non-functional adenoma, with an average tumor volume of $2.9 \pm 4.6 \mathrm{~cm}^{3}$, average follow-up of $72.4 \pm 37.2$ months, and an average dose of $50.4 \mathrm{~Gy}$. The results of those authors showed that the tumor volume of $22.2 \%$ patients decreased, $70.4 \%$ did not change and $7.4 \%$ increased. The 5 -year tumor control rate was estimated to be $95.5 \%$, the 5 -year new tumor survival rate to be $95.8 \%$, and the 5-year visual acuity and pituitary function retention rate to be 93.2 and $95.38 \%$, respectively, indicating that FSRT may be a safe and effective way to treat pituitary macroadenoma. However, their study did not distinguish the specific effects for functional adenoma. Generally, it is held that surgical resection can rapidly relieve the clinical symptoms caused by abnormal hormone of functional adenoma, but is not the preferred method as it is relatively slow with regard to effects (10). Recent findngs suggested that SRT controlled 
the hormone level of functional adenoma in a satisfactory scope (11).

The focus of the present study was on analyzing the specific design regimen and clinical effects of head $\gamma$-SRT in the treatment of functional pituitary macroadenoma, and comparing $\gamma$-SRT using the single nasal-sphenoidal approach. Microsurgical resection has been recognized as the 'gold standard' for the treatment of pituitary tumors owing to little damage, favorable effects, and few complications (12). After treatment, the tumor volumes of patients in the radiotherapy group that received single and multiple irradiation, and the surgery group were significantly reduced and visual acuity and visual field were improved, with differences being statistically significant. Differences in the tumor control, mortality and hypopituitarism rates of the two groups were not statistically significant $(p>0.05)$. The incidence of complication of the radiotherapy group was significantly lower than that of the surgery group, with differences being statistically significant $(\mathrm{p}<0.05)$.

Concerning single or multiple irridiation, Shigematsu et al and Hoban et al suggested that compared with single irradiation, multiple irradiation could better protect the normal tissues $(13,14)$. Loeffler and Shih conducted a meta-analysis and suggested that, multiple irradiation reduced damages to structures sensitive to peripheral X-ray while single irradiation was able to achieve rapid biological remission through a one-time precise strike (15). The accumulation of clinical experience may lead to a more favorable irradiation scheme being developed. MRI, due to its high spatial resolution and soft tissue resolution and its ability to avoid the effects of bone artifacts and support the three-dimensional, multi-angle, multi-parameter scan, may demonstrate the size and shape of pituitary body as well as the location of pituitary stalk, observe the relationship between tumor and optic chiasma, cavernous sinus and surrounding tissues, thereby ensuring a good curative effect (16)

Full attention was paid to reducing the error of positioning and treatment, improving the formability of the target area, improving the dose within the target area, and reducing the dose outside the target area as far as possible. Hypopituitarism is the major complication of radiotherapy, the pituitary - GH shaft is the most sensitive, and the direct risk of GH deficiency is the dose and time of radiotherapy, which may increase as follow-up continues. Thus, the risk factors include radiotherapy dose, single dose, age, irradiation volume of pituitary axis and radiotherapy (17).

In conclusion, $\gamma$-SRT was found to be safe and effective for the treatment of functional pituitary adenomas. Its curative effects were equivalent to that of the microscopic nasalsphenoidal approach although with fewer complications.

\section{References}

1. Laws ER, Sheehan JP, Sheehan JM, Jagnathan J, Jane JA Jr and Oskouian R: Stereotactic radiosurgery for pituitary adenomas: a review of the literature. J Neurooncol 69: 257-272, 2004.

2. Huang BT, Lu JY, Lin PX, Chen JZ, Li DR and Chen CZ: Radiobiological modeling analysis of the optimal fraction scheme in patients with peripheral non-small cell lung cancer undergoing stereotactic body radiotherapy. Sci Rep 5: 18010, 2015.

3. Gopalan R, Schlesinger D, Vance ML, Laws E and Sheehan J: Long-term outcomes after Gamma Knife radiosurgery for patients with a nonfunctioning pituitary adenoma. Neurosurgery 69: 284-293, 2011.

4. Stafford SL, Pollock BE, Leavitt JA, Foote RL, Brown PD, Link MJ, Gorman DA and Schomberg PJ: A study on the radiation tolerance of the optic nerves and chiasm after stereotactic radiosurgery. Int J Radiat Oncol Biol Phys 55: 1177-1181, 2003.

5. Kopp C, Theodorou M, Poullos N, Astner ST, Geinitz H, Stalla GK, Meyer B, Molls M, Nieder C and Grosu AL: Fractionated stereotactic radiotherapy in the treatment of pituitary adenomas. Strahlenther Onkol 189: 932-937, 2013.

6. Senior BA, Ebert CS, Bednarski KK, Bassim MK, Younes M, Sigounas D and Ewend MG: Minimally invasive pituitary surgery. Laryngoscope 118: 1842-1855, 2008.

7. Leksell L: The stereotaxic method and radiosurgery of the brain. Acta Chir Scand 102: 316-319, 1951.

8. Barber SM, Teh BS and Baskin DS: Fractionated stereotactic radiotherapy for pituitary adenomas: single-center experience in 75 consecutive patients. Neurosurgery 9: 1-3, 2015.

9. Weber DC, Momjian S, Pralong FP, Meyer P, Villemure JG and Pica A: Adjuvant or radical fractionated stereotactic radiotherapy for patients with pituitary functional and nonfunctional macroadenoma. Radiat Oncol 6: 169, 2011.

10. Kajiwara K, Saito K, Yoshikawa K, Ideguchi M, Nomura S, Fujii M and Suzuki M: Stereotactic radiosurgery/radiotherapy for pituitary adenomas: A review of recent literature. Neurol Med Chir (Tokyo) 50: 749-755, 2010.

11. Wilson PJ, Williams JR and Smee RI: Cushing's disease: a single centre's experience using the linear accelerator (LINAC) for stereotactic radiosurgery and fractionated stereotactic radiotherapy. J Clin Neurosci 21: 100-106, 2014.

12. Sheehan JM, Sheehan JP, Douds GL and Page RB: DDAVP use in patients undergoing transsphenoidal surgery for pituitary adenomas. Acta Neurochir (Wien) 148: 287-291, discussion 291, 2006.

13. Shigematsu N, Kunieda E, Kawaguchi O, Takeda A, Ihara N, Yamashita S, Kubo A and Ito H; Naoyuki Shigematsu, Etsuo Kunieda: Indications of stereotactic irradiation for brain lesions. Acta Oncol 39: 597-603, 2000.

14. Hoban PW, Jones LC and Clark BG: Modeling late effects in hypofractionated stereotactic radiotherapy. Int J Radiat Oncol Biol Phys 43: 199-210, 1999.

15. Loeffler JS and Shih HA: Radiation therapy in the management of pituitary adenomas. J Clin Endocrinol Metab 96: 1992-2003, 2011.

16. Minniti G, Scaringi C, Poggi M, Jaffrain Rea ML, Trillò G, Esposito V, Bozzao A, Enrici MM, Toscano V and Enrici RM: Fractionated stereotactic radiotherapy for large and invasive non-functioning pituitary adenomas: long-term clinical outcomes and volumetric MRI assessment of tumor response. Eur J Endocrinol 172: 433-441, 2015.

17. Fernandez A, Brada M, Zabuliene L, Karavitaki N and Wass JA: Radiation-induced hypopituitarism. Endocr Relat Cancer 16: 733-772, 2009. doi: 10.1677/ERC-08-0231. 